\title{
The Pellino family: IRAK E3 ligases with emerging roles in innate immune signalling
}

\author{
Paul N. Moynagh \\ Institute of Immunology, National University of Ireland Maynooth, Maynooth, Co. Kildare, Ireland
}

\begin{abstract}
This review highlights the emerging roles of the Pellino family of E3 ubiquitin ligases as upstream mediators in Toll-like receptor (TLR) pathways that lead to activation of MAP kinases and transcription factors. The functional importance of the Pellino family as RING-like-domaincontaining proteins with intrinsic ubiquitin E3 ligase activity that can catalyse polyubiquitylation of the key TLR signalling molecule IRAK1 is discussed in detail. The importance of Pellino proteins as novel targets for mediating negative regulation of TLR signalling is also explored. This new knowledge and understanding of Pellino biology begins to fill some long-standing voids in our understanding of TLR signalling.
\end{abstract}

\section{Introduction}

Toll-like receptors (TLRs) function as primary sensors of conserved microbial structures known as pathogen-associated molecular patterns (PAMPs) [1]. Ten TLRs have been identified in humans, each representing a transmembrane protein with an ectodomain comprising leucine-rich repeats and a cytoplasmic Toll/interleukin (IL)-1 receptor (TIR) domain. TLRs 1, 2, 4, 5 and 6 are expressed on the cell surface and their ectodomains bind PAMPs such as lipopolysaccharide (LPS) and flagellin, which are exposed on the exterior of the microbe. Other TLRs, such as TLR3, TLR7, TLR8 and TLR9, are found in intracellular endosomal compartments in which they serve to detect nucleic acids from invading microbes. The engagement of TLR ectodomains by cognate ligands leads to the recruitment of TIR-domain-containing adaptor proteins to the cytoplasmic TIR domains of TLRs. This triggers activation of transcription factors, such as nuclear factor (NF)- $\mathrm{kB}$ and interferon regulatory factors (IRFs), and mitogen-activated protein kinase (MAPK) cascades. These pathways work in a highly co-ordinated fashion to promote expression of gene profiles that are tailored towards efficient removal of the invading microbe.

The importance of Pellino proteins for TLR signalling Much research effort has focused on the signal transduction cascades that are employed by TLRs in activating transcription factors and MAPK pathways. Ubiquitylation, a form of post-translational modification, is crucially important in the regulation of proteins in these pathways and there is much interest in identifying the enzymes that control such

Corresponding author: Moynagh, P.N. (Paul.Moynagh@nuim.ie). ubiquitylation. The recent discovery of Pellino proteins as a family of E3 ubiquitin ligases that catalyse polyubiquitylation of interleukin-1 receptor-associated kinase (IRAK) molecules in the TLR signalling pathways and regulate activation of NF- $\mathrm{KB}$ and MAPK cascades provides fascinating insight into a new family of signalling molecules in TLR biology. The three mammalian Pellino proteins are highly similar in primary structure (Figure 1a), with each protein possessing a C-terminal RING-like domain that confers E3 ubiquitin ligase activity and an ability to promote polyubiquitylation of IRAK-1. Pellino proteins also contain a phospho-threonine-binding forkhead-associated (FHA) domain that might facilitate Pellino-IRAK-1 interactions (Figure 1b). Pellino proteins can also interact with other TLR signalling molecules, such as TNF receptor-associated factor 6 (TRAF6) and transforming growth factor (TGF)- $\beta$ activated kinase 1 (TAK1), and the formation of such complexes, coupled with Pellino-mediated polyubiquitylation of IRAK1, is probably a key upstream event in TLR signal transduction cascades. This review aims to provide a comprehensive overview of the molecular and functional roles of Pellino proteins in TLR biology, including detailed discussion of the emerging theme of Pellino proteins as targets for strategies to negatively regulate TLR signalling. Given the role of Pellino proteins in regulating NF- $\mathrm{KB}$ and MAPKs, an initial overview of TLR signalling in the context of activating these pathways serves as a valuable framework to discuss the functional roles of the Pellino family.

\section{Overview of TIR-mediated activation of NF-KB and MAPKs TLRs and activation of NF- $\kappa B$}

The cytoplasmic TIR domain of the TLRs is key to initiating all TLR signal transduction cascades. This domain is also found in the IL-1 receptor (IL-1R) [2]. The engagement of the ectodomains of TLRs and IL-1R by their cognate ligands leads to recruitment of soluble TIR-domain-containing adaptor proteins to their cytoplasmic domains. With the exception of TLR3, all TLRs and IL-1R recruit the TIR adaptor myeloid differentiation factor 88 (MyD88) [3]. Although TLR3 and TLR4 can use other MyD88independent pathways (for a review, see Ref. [4]), this review focuses on MyD88-dependent signalling cascades (Figure 2) given that research has largely highlighted Pellino function in MyD88 pathways.

The associations of MyD88 with TLRs and IL-1R are mediated by homotypic TIR-TIR interactions. MyD88 also 


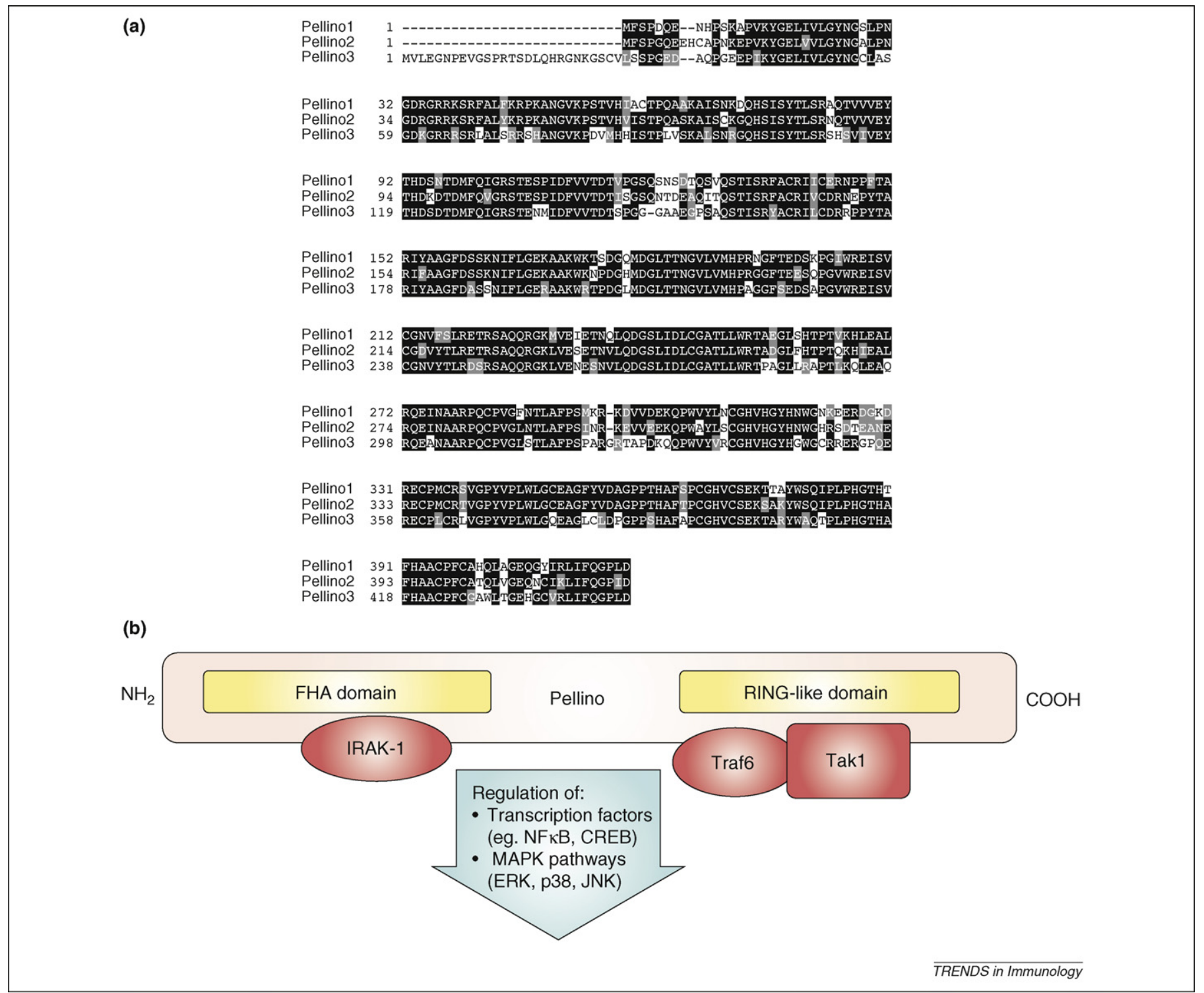

Figure 1. Structure-function characteristics of mammalian Pellino proteins. (a) An alignment of the amino acid sequences of human Pellino1, 2 and 3 is indicated. Sequence identity is represented by black shading and sequence similarity by grey shading. (b) Each Pellino protein has a C-terminal RING-like domain that confers E3 ubiquitin ligase activity. Emerging reports indicate that Pellino2 contains a phosphothreonine-binding FHA domain in its $\mathrm{N}$ terminus that might facilitate interaction with phosphorylated

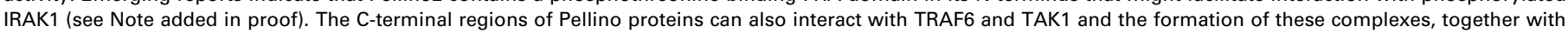
Pellino-induced polyubiquitylation of IRAK-1, regulates activation of transcription factors and MAPK pathways.

contains a death domain that facilitates the subsequent recruitment of death-domain-containing IRAK1 and IRAK4 to the receptor complex [2] where IRAK1 is phosphorylated and activated by IRAK4 [5-7]. This is followed by intensive autophosphorylation of IRAK1 [5,6], its interaction with TRAF6 and subsequent dissociation of IRAK1 and TRAF6 as binding partners from the receptor complex [8-10]. TRAF6 then interacts with TIFA (TRAF-interacting protein with a FHA domain), which promotes oligomerization and ubiquitylation (Box 1) of the former [11]. In this process, TRAF6 interacts with the E2 ubiquitin-conjugating heterodimer UbcH13-Uev1a, resulting in covalent attachment of Lys63-linked polyubiquitin chains to TRAF6 [12]. The IRAK1-TRAF6 complex interacts with another membrane complex consisting of TAK1 and its pre-associated proteins, TAK1-binding protein (TAB)1, TAB2 and TAB3 [13,14], through the recognition of polyubiquitin chains on TRAF6 by highly conserved zinc finger domains in TAB2 and TAB3 [15] (Figure 2). Other reports indicate that TAB2 can interact with unmodified TRAF6 [16] and facilitate its ubiquitylation [17]. TAK1 and TAB2 are subsequently phosphorylated as part of the membrane complex and this is followed by translocation of a putative TRAF6-TAK1TAB1-TAB2-TAB3 complex to the cytosol, at which TAK1 becomes activated by polyubiquitylated TRAF6 $[10,13]$. By contrast, IRAK1 remains in the membrane and is subsequently degraded with kinetics that vary with different TLR stimuli $[10,18,19]$. Active TAK1 promotes downstream activation of the IкB-kinases (IKKs), IKK $\alpha$ and IKK $\beta[13,20]$. The IKKs phosphorylate the NF-KB inhibitory I $\mathrm{KB}$ proteins, leading to polyubiquitylation and, ultimately, degradation of the latter by the $26 \mathrm{~S}$ proteasome [21]. This facilitates the nuclear translocation of NF-кB and induction of a wide range of TLR-responsive genes [22,23]. 


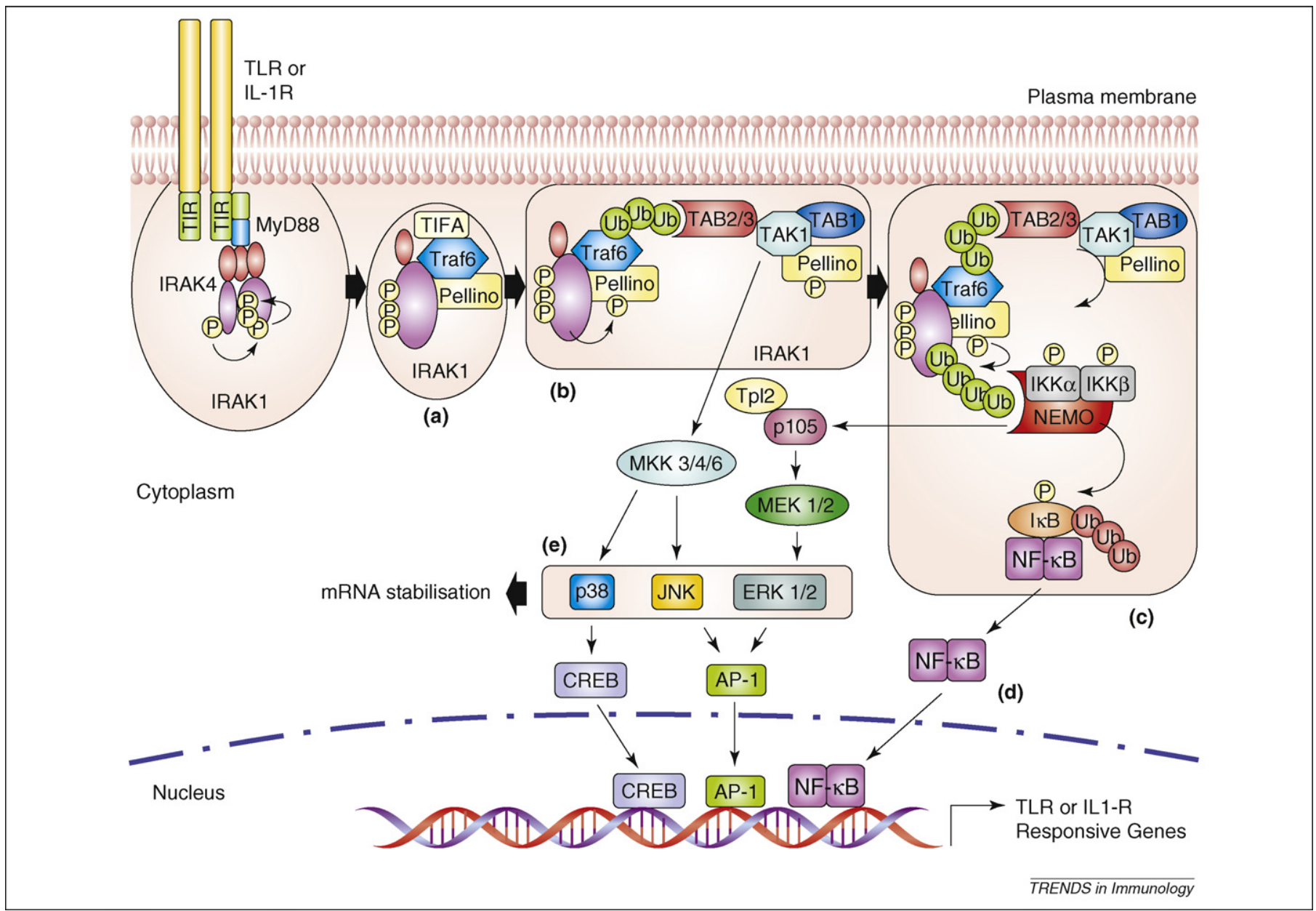

Figure 2. Proposed integration of Pellino proteins in the context of TLR- and IL-1R-dependent activation of NF-kB and MAPK pathways. The figure integrates the findings from several independent studies into a working model that illustrates the likely molecular roles of Pellino proteins in TLR and IL-1R signalling pathways. It should be noted that some of the large protein complexes and connecting steps remain to be experimentally confirmed but are consistent with data published thus far. The working model proposes the following sequence of events: (a) the stimulation of TLRs or IL-1R promotes the association of Pellino proteins with IRAK1 and TRAF6. The K63-linked polyubiquitylation (Ub) of TRAF6 facilitates recruitment and activation of a pre-associated TAK1-TAB complex; (b) IRAK1 phosphorylates (P) Pellino proteins, leading to activation of their E3 ligase activity and Pellino-mediated K63-linked polyubiquitylation of IRAK1; (c) The ubiquitin-binding domain of NEMO binds to polyubiquitylated IRAK1, bringing the IKK and TAK1 complexes into close proximity; (d) TAK1 activates the IKKs, resulting in the phosphorylation (P) of the inhibitory IKB proteins and enabling for translocation of NF- $\mathrm{KB}$ into the nucleus; (e) IKKs also phosphorylate $\mathrm{p} 105$, leading to its degradation and the subsequent release of active Tpl2 that triggers the ERK1/2 MAPK cascade. In addition to interacting with and activating the IKK complex, TAK1 can also trigger the p38 and JNK MAPK pathways via activating the upstream MKKs3, 4 and 6. The MAPK pathways activate several transcription factors that co-operate with NF-KB to induce the expression of a range of TLR-responsive genes. MAPK pathways can also regulate the transport and stability of newly transcribed transcripts.

\section{TLRs and MAPK pathways}

Interestingly, the IKK complex also phosphorylates the $\mathrm{NF}-\mathrm{\kappa B}$ protein $\mathrm{p} 105$, a negative regulator of the serine/ threonine kinase tumour progression locus 2 (TPL-2; also known as Cot) [24]. The phosphorylation of p105 leads to its ubiquitylation and degradation, freeing active TPL-2 to stimulate the classical MAPKs, extracellular signalregulated kinase (ERK)-1 and ERK-2. TLRs can also activate the p38 and JNK MAPK cascades. Active TAK1 phosphorylates MAPK kinase kinases (MKK) $3 / 6$ and

\section{Box 1. The ubiquitylation pathway}

Ubiquitin is a 76 amino acid protein that can be covalently attached to lysine $(\mathrm{K})$ residues of target proteins by a process known as ubiquitylation [60]. This post-translational modification requires the sequential actions of three enzymes. First, ubiquitin is activated by a ubiquitin-activating enzyme (E1), resulting in ATP-dependent thioester formation between the $C$ terminus of ubiquitin and an $E 1$ activesite cysteine. This is followed by the transfer of ubiquitin to the active site cysteine of a ubiquitin-conjugating (Ubc) enzyme (E2). In the final step, a ubiquitin-protein ligase (E3) transfers ubiquitin from E2 and attaches it to a target protein through the formation of an isopeptide bond between the $\mathrm{C}$ terminus of ubiquitin and the $\varepsilon$ amino group of a lysine amino acid in the target protein. The covalent attachment of a single ubiquitin molecule to a target protein is known as monoubiquitylation. However, ubiquitin has seven lysine residues that can also be subjected to ubiquitylation. This can lead to the generation of ubiquitin chains in a process termed polyubiquitylation. The type of lysine linkage that links the polyubiquitin chains is of great functional importance. Polyubiquitin chains that are linked through K48 of ubiquitin usually tag the target protein for degradation by the proteasome. By contrast, polyubiquitin chains that are linked through $\mathrm{K} 63$ of ubiquitin can regulate the structure and function of the target proteins and can impact a diverse range of processes in the immune system, including regulation of gene expression $[61,62]$. 
MKK4 that, in turn, activate p38 and Jun N-terminal kinase (JNK), respectively [13]. Thus, TAK1 emerges as a central player in TLR regulation of NF- $\mathrm{kB}$ and MAPKs, given its ability to activate the IKKs and MKK3, 4 and 6 (Figure 2). However, it should be noted that some reports indicate that TLRs and IL-1R might activate these pathways in a TAK1-independent manner. Thus, in mouse embryonic fibroblasts that lack TAB1, IL-1 is incapable of activating TAK1, yet can still activate IKKs and the p38 and JNK MAPK pathways [25]. The mixed lineage kinases (MLK)-2 and MLK-3 might substitute for TAK1, at least with respect to activating the p38 and JNK MAPKs [26,27]. Irrespective of the exact mechanism, the MAPK pathways can promote activation of the transcription factors cAMP response element-binding (CREB) and activator protein 1 (AP-1) that cooperate with NF-кB at various gene promoters to increase gene transcription [28]. Furthermore, MAPKs can regulate the expression of these genes by post-transcriptional mechanisms, including promoting the nucleocytoplasmic transport and increasing the stability of newly transcribed transcripts.

\section{IRAK polyubiquitylation and TLR/IL-1R signalling}

Although TAK1 promotes phosphorylation and activation of IKK $\alpha$ and IKK $\beta[13,20]$, the exact mechanism by which TAK1 and IKKs are brought into close proximity is not clearly understood. The above-mentioned model favours the dissociation of the multi-molecular TRAF6-TAK1TAB1-TAB2-TAB3 complex from IRAK1 at the membrane followed by its translocation to the cytosol, at which TAK1 becomes active, TAB2 promotes assembly of TRAF 6 with the IKKs and TAK1 activates the IKKs [17]. Thus, IRAK1 remains at the membrane and the activation and assembly of TAK and the IKKs are spatially separate from IRAK1.

However, an alternative model has been proposed based on very recent studies characterizing the ubiquitylation of IRAK1. It is well known that LPS and IL-1 induce the polyubiquitylation and degradation of IRAK1 and it was previously assumed that IRAK1 was modified by K48linked polyubiquitylation that tagged it for proteolysis by the proteasome. However, two recent reports show that TLR4 and IL-1R signalling leads to K63-linked and not K48-linked polyubiquitylation of IRAK1 [16,29], which serves not to tag IRAK for degradation by the proteasome but rather has an important role in TLR- or IL-1Rmediated activation of NF-кB. The studies show that polyubiquitylated IRAK recruits an IKK complex containing catalytic IKK $\alpha$ and IKK $\beta$ in association with their regulatory subunit NF- $\mathrm{KB}$ essential modifier (NEMO; also known as IKK $\gamma$ ). NEMO lacks catalytic activity but is essential for activation of NF-кB [30]. It contains a ubiquitin-binding domain that specifically recognizes K63-linked IRAK1, thus, facilitating IKK-IRAK complex formation as part of TLR and IL-1R signalling [31,32]. IRAK is ubiquitylated on Lys134 and Lys180 and mutation of these sites impairs TLR- or IL-1R-mediated ubiquitylation of IRAK1, binding of IRAK to NEMO and NF-KB activation [29]. These studies indicate a model in which TAK1 is recruited to K63-linked polyubiquitin chains on TRAF6 and the IKK complex is recruited to K63-linked polyubiquitin chains on
IRAK1. This brings TAK1 and IKKs into close proximity, leading to TAK1-mediated phosphorylation and activation of the IKKs. Given this model, there is currently intense interest in identifying the E3 ubiquitin ligase(s) that catalyses polyubiquitylation of IRAK1. It has been proposed [29] that there is, at least, a partial involvement of TRAF6 in the polyubiquitylation of IRAK1, but this has been opposed by two other groups [16,33]. Although the role of TRAF6 as the E3 ligase for IRAK1 remains ambiguous, the existence of a non-TRAF6 E3 ligase(s) that is capable of catalysing K63-linked polyubiquitylation of IRAK1 is widely agreed upon. Pellino proteins emerge as likely candidates to fill this role given their intrinsic E3 ubiquitin ligase activity and their ability to interact with IRAK1.

\section{Bi-directional communication in the IRAK-Pellino complex}

Pellino was first identified in Drosophila melanogaster by virtue of its association with Pelle, the Drosophila orthologue of IRAK [34]. Three mammalian homologues (Pellino1, 2 and 3) have since been identified, with Pellino3 being expressed in two spliced forms [35-39]. Given that Drosophila Pellino was discovered based on its interaction with Pelle, mammalian Pellino proteins have been characterized by their potential to interact with IRAK molecules. Overexpression studies have shown that mammalian Pellino proteins interact with both IRAK1 and IRAK4 [36,37,40-43]. Furthermore, a yeast two-hybrid screen using IRAK4 as bait independently identified Pellino2 as a protein-binding partner [40]. Under physiological conditions, the Pellino-IRAK interactions are likely to be dependent on prior TLR or IL-1R stimulation because endogenous IRAK-Pellino associations are not observed in resting cells but manifest in response to stimulation by IL-1 [33,35-37,44] and LPS [44].

\section{IRAK kinase activity and Pellino binding}

Although not a substrate for the kinase activity of Pelle, Drosophila Pellino can only interact with the catalytically active form of Pelle, indicating that it recognizes autophosphorylated Pelle [34]. Presently, there is disagreement on the importance of IRAK kinase activity for IRAKPellino associations. Some groups have shown that IRAK kinase activity is dispensable for IRAK-Pellino interactions $[40,42,45]$. However, two other groups propose that IRAK1-Pellino binding requires IRAK1 kinase activity $[37,46]$, which is supported by the fact that IRAK1b, a naturally occurring alternative splice variant of IRAK1 that is kinase inactive, fails to interact with Pellino3 [37]. The basis to the opposing conclusions might be a result of interpretations being made exclusively on the basis of overexpression studies. Future studies that characterize endogenous associations of Pellino family members with naturally occurring catalytically inactive members of the IRAK family (e.g. IRAK1b, IRAK2 and IRAK-M) might resolve some of the confusion. It is interesting to note that a very recent report indicates that Pellino proteins have a cryptic FHA domain that mediates interaction with phosphorylated IRAK1 (see Note added in proof). Given that IRAK undergoes 
autophosphorylation, this study would favour a role for IRAK1 kinase activity in promoting IRAK1-Pellino interactions.

\section{IRAKs phosphorylate and enhance E3 ligase activity of Pellino proteins}

The association of Pellino proteins with IRAKs promoted interest in the possibility of Pellino proteins being substrates for IRAK kinase activity. The co-expression of IRAK1 and Pellino proteins leads to phosphorylation of each of the Pellino family [43], with in vitro kinase assays demonstrating that IRAK1 can directly phosphorylate Pellino proteins $[40,45]$. These studies also show that Pellino1 and Pellino2 are substrates for IRAK4. An appreciation of the consequence of IRAK-mediated phosphorylation of the Pellino proteins was aided by an insight into the functional properties of the Pellino family. A bioinformatic analysis had identified a RING-like domain (Box 2) in the C termini regions of the Pellino proteins, indicating that they might function as E3 ubiquitin ligases [38]. This was supported by a study showing that coproduction of Pellino1 or Pellino2, but not Pellino3, with IRAK leads to IRAK polyubiquitylation [43]. Interestingly, two independent groups have shown that all three Pellino proteins, including both splice variants of Pellino3, can induce intense polyubiquitylation of co-expressed IRAK1 [33,42]. In vitro ubiquitylation assays have shown that recombinant and endogenous forms of Pellino1-3 possess E3 ligase activity and, thus, Pellino proteins are likely to directly catalyse polyubiquitylation of IRAK1 $[33,42,45]$. Intriguingly, in an in vitro setting, the Pellino proteins are capable of catalysing the formation of K63- and K48-linked polyubiquitin chains. The type of linkage is dictated by the E2 enzyme that collaborates with the Pellino E3 ligase to catalyse polyubiquitylation. Each of the Pellino proteins can combine with the E2 heterodimer UbcH13-Uev1a to

\section{Box 2. The RING domain}

The classical RING-finger domain is characterized by a highly conserved linear pattern of cysteine and histidine residues: Cys$\mathrm{X}_{2}$-Cys- $\mathrm{X}_{9-39}-\mathrm{Cys}-\mathrm{X}_{1-3}-\mathrm{His}-\mathrm{X}_{2-3}-\mathrm{Cys} / \mathrm{His}-\mathrm{X}_{2}-\mathrm{Cys}-\mathrm{X}_{4-48}-\mathrm{Cys}_{\mathrm{s}} \mathrm{X}_{2}-\mathrm{Cys}$ [63]. The $\mathrm{C} 3 \mathrm{HC} 4$ pattern defines a domain that can fold into a crossbrace structure that is capable of binding two zinc atoms. This RINGfinger domain is usually a signature of proteins that have E3 ubiquitin ligase activity [64]. Intriguingly, Pellino proteins have a pattern of cysteine and histidine residues that is highly related to the RING domain. This RING-like domain in the Pellino family has the pattern Cys- $\mathrm{X}_{1}-\mathrm{His}-\mathrm{X}_{19}$-Cys- $\mathrm{X}_{2}-\mathrm{Cys}_{\mathrm{s}} \mathrm{X}_{30}-\mathrm{Cys}-\mathrm{X}_{1}-\mathrm{His}-\mathrm{X}_{25}-\mathrm{Cys}-\mathrm{X}_{2}-\mathrm{Cys}$ and is abbreviated to $\mathrm{CHC} 2 \mathrm{CHC} 2$ [43].

catalyse K63-linked ubiquitylation. By contrast, detailed studies using mass spectrometry show that Pellino1 can combine with UbcH3 to catalyse formation of K48-linked polyubiquitin chains, whereas, in combination with UbcH4, UbcH5a or UbcH5b, it promotes formation of K48- and K11-linked polyubiquitin chains [45]. However, cellular studies to date indicate that the co-expression of Pellino proteins with IRAK1 induces only K63-linked polyubiquitylation of IRAK1 $[33,45]$. It is possible that Pellino proteins might be subject to regulatory processes in vivo that facilitate their interaction with different E2 enzymes and, hence, confer the ability to promote the formation of polyubiquitin chains that are linked by other lysine residues.

It has already been mentioned that Pellino proteins are phosphorylated by IRAK1 and IRAK4 and the functional consequence of this phosphorylation is enhancement of Pellino E3 ligase activity [45]. A plausible model emerges in which TLRs or IL-1R promote IRAK-Pellino interactions, leading to IRAK-mediated phosphorylation of the Pellino proteins, enhancement of their E3 ligase activity and, ultimately, Pellino-mediated polyubiquitylation of the IRAK1 (Figure 3). Indeed, the suppression of

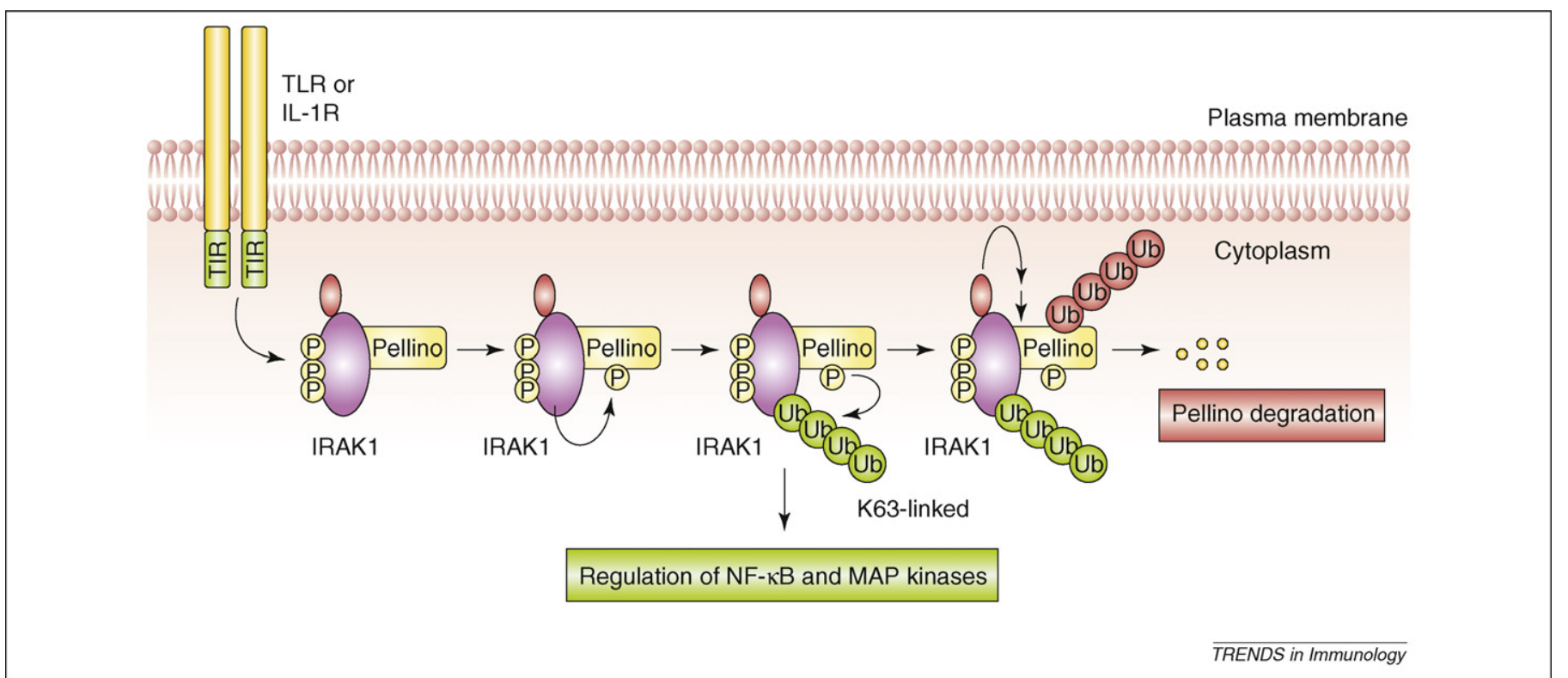

Figure 3. Bi-directional communication between IRAKs and Pellino proteins. The stimulation of TLRs or IL-1R promotes the association of Pellino proteins with IRAK1. The latter phosphorylates the Pellino proteins, leading to activation of their E3 ligase activity. This leads to Pellino-mediated formation of K63-linked polyubiquitin chains on IRAK. Polyubiquitylated IRAK can regulate several downstream pathways, including NF-KB and MAPK cascades. Kinase active IRAK can also promote polyubiquitylation of the Pellino proteins by an unknown mechanism, resulting in Pellino degradation. 
endogenous Pellino3 by siRNAs causes a considerable reduction of IL-1-induced polyubiquitylation of IRAK1 [33].

\section{Kinase-active IRAKs promote polyubiquitylation and degradation of Pellino proteins}

Interestingly, the IRAK-Pellino interactions also lead to reciprocal polyubiquitylation of the Pellino-binding partners [42] (Figure 3). The co-expression of kinase-active IRAK1 or IRAK4 with Pellino proteins causes intense polyubiquitylation of Pellino family members. The identity of the E3 ligase(s) that catalyses the IRAK-induced polyubiquitylation of the Pellino family remains to be elucidated, with reports differing on the relevant importance of Pellino E3 ligase activity in this process $[42,45]$. The polyubiquitylation of Pellino proteins is associated with subsequent Pellino degradation. This is a potential mechanism for regulating TLR signalling and, indeed, the TLR4 ligand LPS induces degradation of Pellino3 in peripheral blood mononuclear cells [42]. Thus, a complex model of bi-directional communication is now emerging with respect to IRAK-Pellino associations, in which IRAKs phosphorylate and activate the Pellino proteins, leading to polyubiquitylation of both binding partners (Figure 3 ). The polyubiquitylation of Pellino proteins leads to their degradation and might be a means to terminate TLR signalling, whereas the K63-linked polyubiquitylation of IRAK is probably an important step in triggering downstream signalling (Figure 3). Indeed, as stated previously, a currently favoured model for MyD88-dependent TLR signalling favours a scenario in which TAK1 is recruited to K63linked polyubiquitin chains on TRAF6 and the IKK complex is recruited to K63-linked polyubiquitin chains on IRAK1. An important role for the E3 ligase activity of Pellino proteins in this model is supported by the finding that wild-type Pellino2, but not a point-mutated form that lacks a functional RING domain, promotes the recruitment of NEMO to K63-linked polyubiquitylated IRAK [45].

\section{Pellino proteins interact with other TLR and IL1-R signalling proteins}

Pellino proteins can also interact with other TLR signalling molecules. None of the Pellino proteins have been reported to interact with TLR or IL-1R complexes, however, one report indicates that IL-1 signalling can promote the interaction of overexpressed Pellino1 with MyD88 [44]. However, other reports have failed to detect any basal interactions between overexpressed MyD88 and Pellino proteins [37,47]. Many groups have described associations of each of the Pellino proteins with TRAF6 and these interactions are strongly promoted by IL-1R signalling [33,36,37,41,44,47]. The nature of these interactions remains to be defined with no available data to date to indicate whether Pellino proteins interact directly with TRAF-6 or indirectly as part of a larger protein complex. Pellino3 has also been reported to interact with TAK1 $[33,37,41]$. Although an early study used overexpression analyses to show that IL-1 can enhance the Pellino3-TAK1 interaction [37], a more recent report provides strong support for a constitutive interaction between Pellino3 and TAK1 [33]. Thus, Pellino3 might be preassociated with the TAK1-TAB complex and is brought into close association with IRAK-1 and TRAF-6 when
IL-1 signalling promotes the association of IRAK1-TRAF6 with the TAK1 complex (Figure 1). This is also consistent with the finding that the IL-1-induced association of TRAF6 with the TAK1 complex and the resulting activation of TAK1 precede the polyubiquitylation of IRAK1 and activation of IKKs [16]. Interestingly, Pellino3 has also been reported to interact with NF-кB-inducing kinase (NIK) [37], a kinase that is involved in the activation of the alternative (NEMOindependent) NF- $\mathrm{KB}$ pathways by receptors, such as the lymphotoxin- $\beta$ receptor [48]. This indicates that Pellino proteins might also serve signalling roles in non-TLR and non-IL-1R pathways.

\section{Functional roles of Pellino proteins in TLR and IL-1R signalling pathways}

The association of Pellino proteins with key signalling molecules in the TLR and IL-1R pathways has prompted characterization of their involvement in mediating downstream signalling cascades. Although each of the Pellino proteins interact with common signalling molecules, such as IRAKs and TRAF6, and each has E3 ubiquitin ligase activity that can promote polyubiquitylation of IRAK1, emerging data clearly show that the Pellino proteins are not functionally redundant but instead display signs of specificity of function.

\section{Pellino 1}

There are contrasting reports on the ability of overexpressed Pellino1 protein to induce a NF-кB-regulated reporter gene $[10,44]$, however, these same groups have shown that suppression of endogenous Pellino1 by siRNAs impairs IL-1induced activation of NF-кB in a human [36] and murine [44] cell line. Furthermore, suppression of Pellino1 strongly inhibits the ability of IL-1 to induce the NF-кB-responsive gene $I L-8$ [36]. Interestingly, Pellino1 is not involved in regulating any of the MAPK pathways $[41,47]$.

\section{Pellino2}

The functional role of Pellino2 in TLR or IL-1R signalling is somewhat controversial. Although overexpression of the Pellino2 protein is incapable of driving expression of a NF-кB-regulated reporter gene, the suppression of Pellino2 in murine embryonic fibroblast or macrophage cell lines inhibits LPS- and IL-1-dependent but not TNF-dependent activation of NF-кB-regulated promoters [35,49]. Overexpression studies have also been used to suggest that Pellino2 drives activation of the ERK1/2 and JNK pathways [47]. However, some data [40] strongly question the specificity of the role of Pellino2 in the NF-кB and MAPK pathways and the above-mentioned conclusions from reporter systems. It has been proposed that many of the findings can be explained by a more generic role for Pellino2 in regulating the general transcription machinery rather than targeting specific pathways. The generation of Pellino2-deficient mice will undoubtedly resolve the present confusion.

\section{Pellino3}

Intriguingly, a recent report has proposed that Pellino3 functions as a negative regulator of TAK1-mediated activation of NF-кB, with the inhibitory effects being dependent on a functional RING domain and on Pellino3-mediated 
K63-linked polyubiquitylation of IRAK1 [33]. The authors propose that IL-1 promotes both K48- and K63-linked polyubiquitylation of IRAK1 and Pellino3-mediated K63-linked IRAK polyubiquitylation competes with K48-linked IRAK polyubiquitylation for the same ubiquitylation site, K134 of IRAK. Thus, Pellino3 blocks IRAK degradation and so inhibits IL-1-induced activation of NF-кB. However, this model requires a positive role for IRAK degradation in IL-1 signalling and requires that IRAK degradation facilitates dissociation of the TAK-1-TAB-TRAF6 complex from the membrane and its subsequent translocation to the cytosol, where it activates the IKK complex [33]. This differs from the model in which K63-linked polyubiquitylated TRAF6 recruits the TAK1 complex and K63-linked polyubiquitylated IRAK1 recruits the IKK complex, thus, bringing TAK1 and IKK into close proximity and enabling IKK activation. Furthermore, in this model, it is proposed that K48-linked polyubiquitylation of IRAK1 leads to its proteasomemediated degradation and Pellino3 prevents this degradation by instead catalysing K63-linked polyubiquitylation of IRAK [33]. This is consistent with a recent independent report demonstrating K48- and K63-linked polyubiquitylation of IRAK1 and proteasome-mediated degradation of IRAK1 in response to IL-1 signalling [50]. However, two other groups contend that blockade of the proteolytic activity of the proteasome fails to prevent IL-1-induced degradation of IRAK1 $[16,29]$ and this questions the importance of K48-linked polyubiquitylation for IRAK degradation. Furthermore, a mutant form of IRAK1 in which Lys134 is mutated to arginine, a residue that is not subject to ubiquitylation, is still capable of triggering activation of $\mathrm{NF}-\kappa \mathrm{B}$ [29]. Interestingly, this study demonstrates that Lys180, as well as Lys134, in IRAK1 is ubiquitylated in response to TLR and IL-1R signalling and the mutation of both residues is required to impair ubiquitylation of IRAK1 and the ability of the latter to activate NF-кB. Although the exact mechanism underlying the inhibitory effects of Pellino3 on the NF-кB pathway remains to be fully delineated and awaits the generation of Pellino3-deficient mice, the proposed negative regulatory role for Pellino3 is a fascinating one and promotes it as an important regulator of TLR and IL-1R pathways.

Although transient overexpression of Pellino3 protein causes activation of the JNK [33,37] and ERK MAPK pathways [37], the suppression of endogenous expression of Pellino3 enhances IL-1-dependent activation of JNK [33], indicating a negative role for Pellino3 in the JNK pathway. However, Pellino3 has a positive role in activation of p38 MAPK and is a mediator in IL-1-dependent activation of p38 [41]. The stimulation of $\mathrm{p} 38$ by Pellino3 is mediated by TRAF 6 and TAK1 and leads to activation of the p38 substrate, MAPK-activated protein kinase 2 . Interestingly, Pellino3 is capable of exhibiting contrasting stimulatory and inhibitory effects on different pathways that are downstream of TAK1. The molecular basis to such complex effects remains to be defined.

\section{Pellino proteins as novel targets for negative regulation of TLR signalling}

The functional characterization of the Pellino family to date highlights their diverse and varying roles in innate immune signalling. Intriguingly, some studies have demonstrated that members of the Pellino family can be targeted by regulatory strategies to suppress TLR signalling [33,44,49]. Indeed, the role of Pellino3 as a direct negative regulator of innate immune signalling has already been described [33] (Figure 4a). However, two additional reports have identified Pellino1 and Pellino2 as novel targets for other inhibitory molecules and pathways [44,49] (Figure $4 b, c)$. TGF- $\beta 1$ has been characterized as an anti-inflammatory cytokine that cross-talks with and downregulates TLR pathways. Thus, TGF- $\beta 1$-deficient mice experience heightened inflammatory responses and early death and this is mainly mediated by abnormal activation of the NF-кB pathway by TLR4 [51,52]. A recent study demonstrated that Pellino1 is targeted by TGF- $\beta 1$ as part of the manifestation of its anti-inflammatory inhibitory effects (Figure $4 \mathrm{~b}$ ). Thus, TGF- $\beta 1$ induces the inhibitory Smad6 protein that subsequently binds to and sequesters Pellino1 and inhibits the ability of Pellino1 to interact with IRAK1, MyD88 and TRAF6 [44]. It is proposed that the disruption of the Pellino1-IRAK-TRAF6 signalling complex by Smad6 is responsible for the inhibitory effects of Smad6 on the LPS and IL-1-stimulated activation of NF-кB and induction of pro-inflammatory genes.

Another study has shown Pellino2 to be targeted by suppressor of cytokine signalling 3 (SOCS3) as part of another mechanism to downregulate TLR signalling [49] (Figure 4c). SOCS3 is induced by LPS, inhibits LPS signalling and is required for the suppressive effects of IL-10 on the TLR4 signalling pathway [53,54]. A recent study identified B-cell lymphoma 10 (BCL10), an intracellular activator of NF- $\mathrm{KB}$, as a binding partner for Pellino2 and proposes the inhibitory effects of SOCS3 to be mediated by blocking the interaction of BCL10 with Pellino2 [49]. In this study, BCL10 interacted with TLR4, associated with Pellino2 and activated the NF-kB pathway in a Pellino2dependent manner. The interaction of SOCS3 with BCL10 blocked the BCL10-Pellino2 association, thereby leading to inhibition of the downstream activation of NF-кB.

The direct role of Pellino3 as a negative regulator of TLR or IL-1R signalling, coupled with the targeting of Pellino1 and Pellino2 by Smad6 and SOCS3, clearly highlights the Pellino family as key modulators of innate immune signalling.

\section{Concluding remarks and future perspectives}

The discovery of the Pellino family has provided new insight into innate immunity and informed an understanding of previously unanswered questions in the field of TLR signalling. The role of the kinase activity of IRAK1 has remained an enigma since its original characterization, but Pellino-related studies now strongly indicate that IRAK1 and/or IRAK4 phosphorylate members of the Pellino family and enhance their E3 ligase activity, leading to polyubiquitylation of IRAK. Furthermore, the kinase activity of IRAK promotes the degradation of Pellino proteins and this might be an important regulatory mechanism that controls TLR signalling.

Although we await the generation of Pellino-deficient mice to define their physiological roles, studies to date 


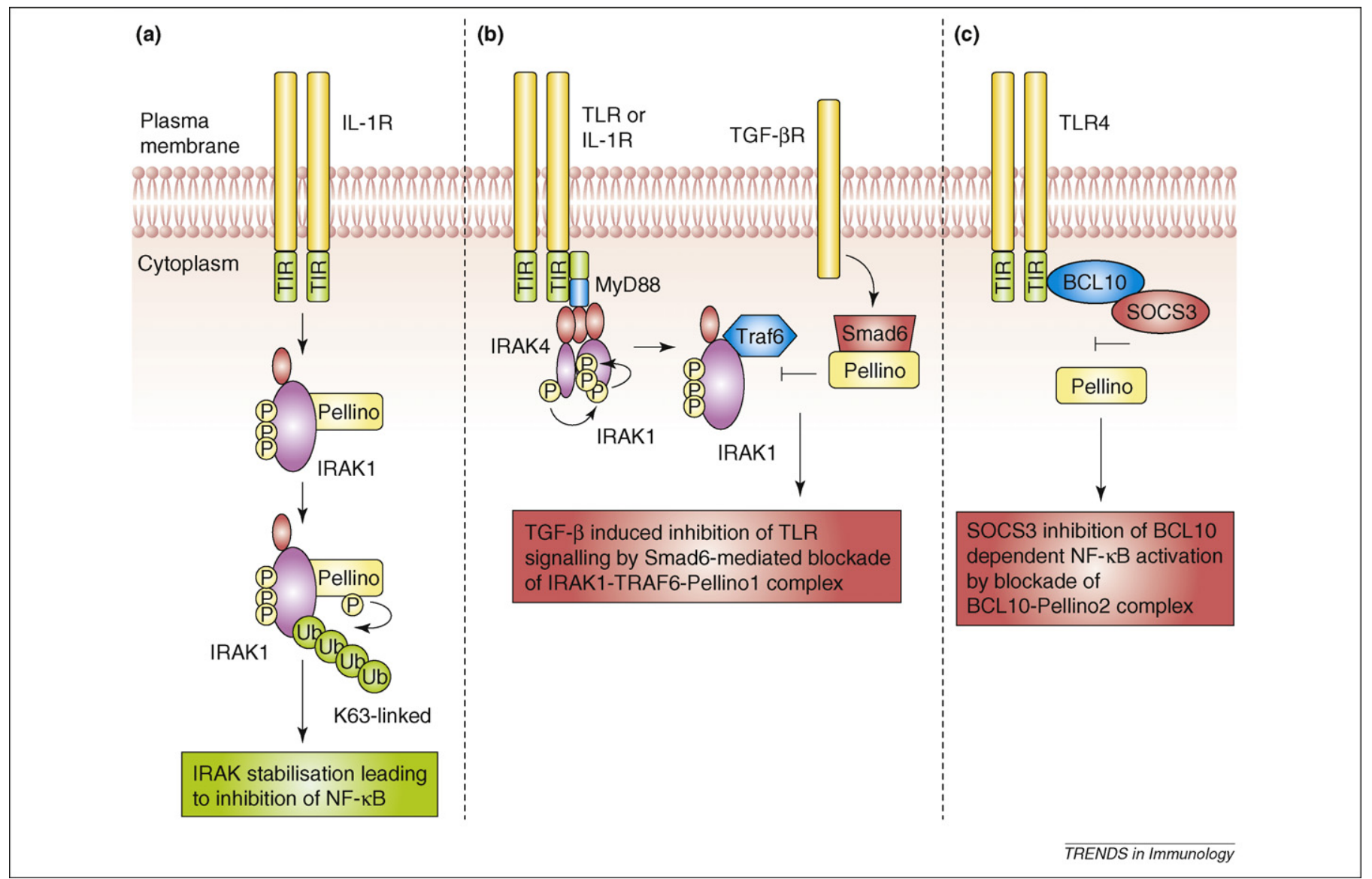

Figure 4. Targeting of Pellino proteins for negative regulation of TLR or IL-1R signalling. Several control systems have been identified that target Pellino proteins to

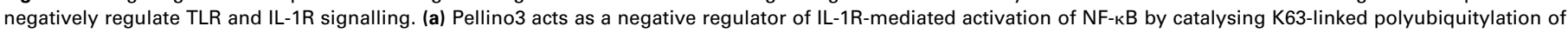
IRAK1, leading to its stabilization. (b) TGF- $\beta$ negatively regulates TLR or IL-1 signalling by inducing Smad6 that subsequently sequesters Pellino1 and, thus, inhibits formation of the IRAK1-TRAF6-Pellino1 complex. This blocks TLR- or IL-1R-induced activation of the NF-KB pathway. (c) BCL10 associates with activated TLR4 and, subsequently, interacts with Pellino2 to activate the NF-KB pathway. SOCS3 is induced by LPS and inhibits the NF-kB pathway by interacting with BCL10 and blocking the BCL10-Pellino association.

support diverse roles for the family with members failing to show functional redundancy. Furthermore, our current knowledge of Pellino biology poses further intriguing questions. Each Pellino member shares molecular characteristics, such as possessing E3 ligase activity, acting as substrates for IRAK kinases and interacting with common signalling molecules, and so we await understanding of the basis to their functional diversity. With this in mind, a detailed understanding of the mechanisms that control Pellino function is required. Indeed, studies have already shown that Pellino proteins are subject to degradation and re-synthesis by TLR signals [42,55,56] and their E3 ligase activity is regulated by IRAK-mediated phosphorylation [45]. This indicates that Pellino proteins are subject to multiple levels of regulation by TLRs, again emphasizing their probable key roles in innate immunity.

Given the role of IRAK1 in promoting activation of IRFs in TLR7 and TLR9 signalling [57], it will be especially interesting to explore the role of Pellino proteins in these viral-sensing pathways. Much effort will also continue to investigate the functional consequence of polyubiquitylation of IRAK1 and probe the physiological importance of Pellino proteins in regulating such modification of IRAK1. Finally, it is worth mentioning that Pellino function might not be restricted to the immune system. A Pellino homologue in Ciona intestinalis (a primitive chordate) has a key role in notochord formation in early development [58,59]. It will be interesting to examine the role of the different Pellino proteins in the development of higher organisms. This is especially intriguing given that Pellino was first characterized in the context of Drosophila Toll signalling and members of the Toll family also exhibit dualist functions in development and the innate immune system. This indicates that the Pellino family has co-evolved closely with the Toll system, supporting the notion of key fundamental roles for Pellino proteins in Toll-like receptor signalling. Although the field of Pellino biology is in its infancy, it represents an area of enormous potential to inform our understanding of the workings of the innate immune system.

\section{Note added in proof}

A study published after this article had been accepted revealed for the first time that Pellino proteins contain a cryptic FHA domain that mediates interaction with phosphorylated IRAK1.

Lin et al., Pellino Proteins Contain a Cryptic FHA Domain that Mediates Interaction with Phosphorylated IRAK1, Structure (2008), doi:10.1016/j.str.2008.09.011 


\section{Acknowledgements}

P.N.M. is funded by Science Foundation Ireland, Health Research Board of Ireland and Enterprise Ireland.

\section{References}

1 Janeway, C.A., Jr and Medzhitov, R. (2002) Innate immune recognition. Annu. Rev. Immunol. 20, 197-216

2 Martin, M.U. and Wesche, H. (2002) Summary and comparison of the signaling mechanisms of the Toll/interleukin-1 receptor family. Biochim. Biophys. Acta 1592, 265-280

3 Medzhitov, R. et al. (1998) MyD88 is an adaptor protein in the hToll/IL1 receptor family signaling pathways. Mol. Cell 2, 253-258

4 O'Neill, L.A. and Bowie, A.G. (2007) The family of five: TIR-domaincontaining adaptors in Toll-like receptor signalling. Nat. Rev. Immunol. 7, 353-364

$5 \mathrm{Li}$, S. et al. (2002) IRAK-4: a novel member of the IRAK family with the properties of an IRAK-kinase. Proc. Natl. Acad. Sci. U. S. A. 99, 55675572

6 Cao, Z. et al. (1996) IRAK: a kinase associated with the interleukin-1 receptor. Science $271,1128-1131$

7 Lye, E. et al. (2004) The role of interleukin 1 receptor-associated kinase4 (IRAK-4) kinase activity in IRAK-4-mediated signaling. J. Biol. Chem. 279, 40653-40658

8 Kollewe, C. et al. (2004) Sequential autophosphorylation steps in the interleukin-1 receptor-associated kinase-1 regulate its availability as an adapter in interleukin-1 signaling. J. Biol. Chem. 279, 52275236

9 Burns, K. et al. (2000) Tollip, a new component of the IL-1RI pathway, links IRAK to the IL-1 receptor. Nat. Cell Biol. 2, 346-351

10 Jiang, Z. et al. (2002) Interleukin-1 (IL-1) receptor-associated kinasedependent IL-1-induced signaling complexes phosphorylate TAK1 and TAB2 at the plasma membrane and activate TAK1 in the cytosol. Mol. Cell. Biol. 22, 7158-7167

11 Takatsuna, H. et al. (2003) Identification of TIFA as an adapter protein that links tumor necrosis factor receptor-associated factor 6 (TRAF6) to interleukin-1 (IL-1) receptor-associated kinase-1 (IRAK-1) in IL-1 receptor signaling. J. Biol. Chem. 278, 12144-12150

12 Deng, L. et al. (2000) Activation of the IкB kinase complex by TRAF6 requires a dimeric ubiquitin-conjugating enzyme complex and a unique polyubiquitin chain. Cell 103, 351-361

13 Wang, C. et al. (2001) TAK1 is a ubiquitin-dependent kinase of MKK and IKK. Nature 412, 346-351

14 Cheung, P.C. et al. (2004) TAB3, a new binding partner of the protein kinase TAK1. Biochem. J. 378, 27-34

15 Kanayama, A. et al. (2004) TAB2 and TAB3 activate the NF-кB pathway through binding to polyubiquitin chains. Mol. Cell 15, 535-548

16 Windheim, M. et al. (2008) Interleukin-1 (IL-1) induces the Lys63linked polyubiquitination of IL-1 receptor-associated kinase 1 to facilitate NEMO binding and the activation of IкB $\alpha$ kinase. Mol. Cell. Biol. 28, 1783-1791

17 Kishida, S. et al. (2005) TAK1-binding protein 2 facilitates ubiquitination of TRAF6 and assembly of TRAF6 with IKK in the IL-1 signaling pathway. Genes Cells 10, 447-454

18 Jacinto, R. et al. (2002) Lipopolysaccharide- and lipoteichoic acidinduced tolerance and cross-tolerance: distinct alterations in IL-1 receptor-associated kinase. J. Immunol. 168, 6136-6141

19 Yamin, T.T. and Miller, D.K. (1997) The interleukin-1 receptorassociated kinase is degraded by proteasomes following its phosphorylation. J. Biol. Chem. 272, 21540-21547

20 Ninomiya-Tsuji, J. et al. (1999) The kinase TAK1 can activate the NIK$\mathrm{I} \kappa \mathrm{B}$ as well as the MAP kinase cascade in the IL-1 signalling pathway. Nature 398, 252-256

$21 \mathrm{Yi}$, A.K. and Krieg, A.M. (1998) CpG DNA rescue from anti-IgMinduced WEHI-231 B lymphoma apoptosis via modulation of IкB $\alpha$ and IкB $\beta$ and sustained activation of nuclear factor-кB/c-Rel. $J$. Immunol. 160, 1240-1245

22 Medzhitov, R. et al. (1997) A human homologue of the Drosophila Toll protein signals activation of adaptive immunity. Nature 388, 394-397

23 O'Neill, L.A. (2002) Signal transduction pathways activated by the IL-1 receptor/toll-like receptor superfamily. Curr. Top. Microbiol. Immunol. $270,47-61$

24 Beinke, S. et al. (2004) Lipopolysaccharide activation of the TPL-2/ MEK/extracellular signal-regulated kinase mitogen-activated protein kinase cascade is regulated by ІкB kinase-induced proteolysis of NFкB1 p105. Mol. Cell. Biol. 24, 9658-9667

25 Mendoza, H. et al. (2008) Roles for TAB1 in regulating the IL-1dependent phosphorylation of the TAB3 regulatory subunit and activity of the TAK1 complex. Biochem. J. 409, 711-722

26 Zhong, J. and Kyriakis, J.M. (2007) Dissection of a signaling pathway by which pathogen-associated molecular patterns recruit the JNK and p38 MAPKs and trigger cytokine release. J. Biol. Chem. 282, 24246-24254

27 Zhong, J. and Kyriakis, J.M. (2004) Germinal center kinase is required for optimal Jun N-terminal kinase activation by Toll-like receptor agonists and is regulated by the ubiquitin proteasome system and agonist-induced, TRAF6-dependent stabilization. Mol. Cell. Biol. 24, 9165-9175

28 Dong, C. et al. (2002) MAP kinases in the immune response. Annu. Rev. Immunol. 20, 55-72

29 Conze, D.B. et al. (2008) Lys63-linked polyubiquitination of IRAK-1 is required for interleukin-1 receptor- and toll-like receptor-mediated NF-кB activation. Mol. Cell. Biol. 28, 3538-3547

30 Rothwarf, D.M. et al. (1998) IKK- $\gamma$ is an essential regulatory subunit of the IкB kinase complex. Nature 395, 297-300

$31 \mathrm{Ea}, \mathrm{C} . \mathrm{K}$. et al. (2006) Activation of IKK by TNF $\alpha$ requires site-specific ubiquitination of RIP1 and polyubiquitin binding by NEMO. Mol. Cell $22,245-257$

$32 \mathrm{Wu}$, C.J. et al. (2006) Sensing of Lys 63 -linked polyubiquitination by NEMO is a key event in NF-кB activation. Nat. Cell Biol. 8, 398-406

$33 \mathrm{Xiao}$, H. et al. (2008) Pellino $3 \mathrm{~b}$ negatively regulates interleukin-1-induced TAK1-dependent NF кB activation. J. Biol. Chem. 283, 14654-14664

34 Grosshans, J. et al. (1999) Oligomerisation of Tube and Pelle leads to nuclear localisation of dorsal. Mech. Dev. 81, 127-138

$35 \mathrm{Yu}, \mathrm{K}$.Y.et al. (2002) Cutting edge: mouse pellino-2 modulates IL-1 and lipopolysaccharide signaling. J. Immunol. 169, 4075-4078

36 Jiang, Z. et al. (2003) Pellino 1 is required for interleukin-1 (IL-1) mediated signaling through its interaction with the IL-1 receptorassociated kinase 4 (IRAK4)-IRAK-tumor necrosis factor receptorassociated factor 6 (TRAF6) complex. J. Biol. Chem. 278, 10952-10956

37 Jensen, L.E. and Whitehead, A.S. (2003) Pellino3, a novel member of the Pellino protein family, promotes activation of c-Jun and Elk-1 and may act as a scaffolding protein. J. Immunol. 171, 1500-1506

38 Rich, T. et al. (2000) Pellino-related sequences from Caenorhabditis elegans and Homo sapiens. Immunogenetics 52, 145-149

39 Resch, K. et al. (2001) Assignment of homologous genes, Peli1/PELI1 and Peli2/PELI2, for the Pelle adaptor protein Pellino to mouse chromosomes 11 and 14 and human chromosomes 2 p13.3 and $14 q 21$, respectively, by physical and radiation hybrid mapping. Cytogenet. Cell Genet. 92, 172-174

40 Strelow, A. et al. (2003) Characterization of Pellino2, a substrate of IRAK1 and IRAK4. FEBS Lett. 547, 157-161

41 Butler, M.P. et al. (2005) Pellino3 is a novel upstream regulator of p38 MAPK and activates CREB in a p38-dependent manner. J. Biol. Chem. 280, 27759-27768

42 Butler, M.P. et al. (2007) Kinase-active interleukin-1 receptorassociated kinases promote polyubiquitination and degradation of the Pellino family: direct evidence for PELLINO proteins being ubiquitin-protein isopeptide ligases. J. Biol. Chem. 282, 29729-29737

43 Schauvliege, R. et al. (2006) Pellino proteins are more than scaffold proteins in TLR/IL-1R signalling: a role as novel RING E3-ubiquitinligases. FEBS Lett. 580, 4697-4702

44 Choi, K.C. et al. (2006) Smad6 negatively regulates interleukin 1receptor-Toll-like receptor signaling through direct interaction with the adaptor Pellino-1. Nat. Immunol. 7, 1057-1065

45 Ordureau, A. et al. (2008) The IRAK-catalysed activation of the E3 ligase function of Pellino isoforms induces the Lys63-linked polyubiquitination of IRAK1. Biochem. J. 409, 43-52

46 Schauvliege, R. et al. (2007) Pellino proteins: novel players in TLR and IL-1R signalling. J. Cell. Mol. Med. 11, 453-461

47 Jensen, L.E. and Whitehead, A.S. (2003) Pellino2 activates the mitogen activated protein kinase pathway. FEBS Lett. 545, 199-202

48 Dejardin, E. et al. (2002) The lymphotoxin- $\beta$ receptor induces different patterns of gene expression via two NF- $\mathrm{KB}$ pathways. Immunity 17 , 525-535

49 Liu, Y. et al. (2004) BCL10 mediates lipopolysaccharide/toll-like receptor-4 signaling through interaction with Pellino2. J. Biol. Chem. 279, 37436-37444 
50 Newton, K. et al. (2008) Ubiquitin chain editing revealed by polyubiquitin linkage-specific antibodies. Cell 134, 668-678

51 Kulkarni, A.B. et al. (1993) Transforming growth factor $\beta 1$ null mutation in mice causes excessive inflammatory response and early death. Proc. Natl. Acad. Sci. U. S. A. 90, 770-774

52 McCartney-Francis, N. et al. (2004) Aberrant Toll receptor expression and endotoxin hypersensitivity in mice lacking a functional TGF- $\beta 1$ signaling pathway. J. Immunol. 172, 3814-3821

53 Berlato, C. et al. (2002) Involvement of suppressor of cytokine signaling-3 as a mediator of the inhibitory effects of IL-10 on lipopolysaccharide-induced macrophage activation. J. Immunol. 168, 6404-6411

54 Stoiber, D. et al. (1999) Lipopolysaccharide induces in macrophages the synthesis of the suppressor of cytokine signaling 3 and suppresses signal transduction in response to the activating factor IFN- $\gamma$. $J$. Immunol. 163, 2640-2647

55 Weighardt, H. et al. (2004) Identification of a TLR4- and TRIFdependent activation program of dendritic cells. Eur. J. Immunol. $34,558-564$
56 Sun, Y. et al. (2008) Toll-like receptor 4 signaling plays a role in triggering periodontal infection. FEMS Immunol. Med. Microbiol. 52, 362-369

57 Moynagh, P.N. (2005) TLR signalling and activation of IRFs: revisiting old friends from the NF-кB pathway. Trends Immunol. 26, 469-476

58 Hotta, K. et al. (2007) Brachyury-downstream notochord genes and convergent extension in Ciona intestinalis embryos. Dev. Growth Differ. 49, 373-382

59 Hotta, K. et al. (2000) Characterization of Brachyury-downstream notochord genes in the Ciona intestinalis embryo. Dev. Biol. 224, 69-80

60 Hershko, A. and Ciechanover, A. (1998) The ubiquitin system. Annu. Rev. Biochem. 67, 425-479

61 Liu, Y.C. et al. (2005) Immunity by ubiquitylation: a reversible process of modification. Nat. Rev. Immunol. 5, 941-952

62 Chen, Z.J. (2005) Ubiquitin signalling in the NF-кB pathway. Nat. Cell Biol. 7, 758-765

63 Freemont, P.S. (2000) RING for destruction? Curr. Biol. 10, R84-R87

64 Joazeiro, C.A. and Weissman, A.M. (2000) RING finger proteins: mediators of ubiquitin ligase activity. Cell 102, 549-552 